Provided for non-commercial research and education use. Not for reproduction, distribution or commercial use.

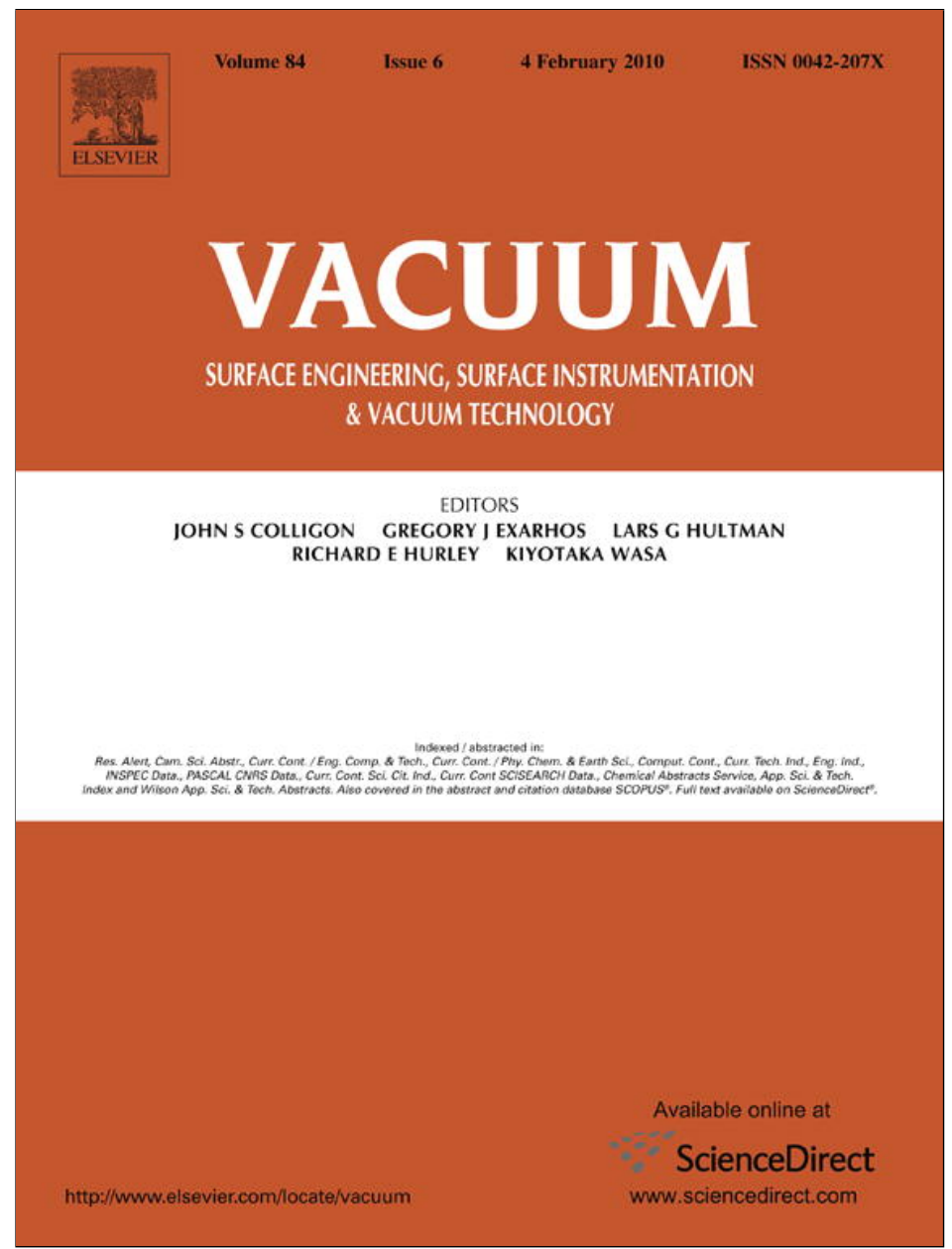

This article appeared in a journal published by Elsevier. The attached copy is furnished to the author for internal non-commercial research and education use, including for instruction at the authors institution and sharing with colleagues.

Other uses, including reproduction and distribution, or selling or licensing copies, or posting to personal, institutional or third party websites are prohibited.

In most cases authors are permitted to post their version of the article (e.g. in Word or Tex form) to their personal website or institutional repository. Authors requiring further information regarding Elsevier's archiving and manuscript policies are encouraged to visit:

http://www.elsevier.com/copyright 


\title{
Radial structure of low pressure rf capacitive discharges
}

\author{
V.A. Lisovskiy*, N.D. Kharchenko, V.D. Yegorenkov \\ Department of Physics and Technology, Kharkov National University, 4 Svobody sq., Kharkov 61077, Ukraine
}

\section{A R T I C L E I N F O}

\section{Article history:}

Received 21 June 2009

Received in revised form

10 September 2009

Accepted 21 October 2009

\section{Keywords:}

Radio frequency discharge

Radial structure

Langmuir probe

Electron energy distribution function

Sheath

\begin{abstract}
A B S T R A C T
This paper studies the glow intensity distribution of the discharge plasma against the tube radius and reports the radial profiles of electron temperature and plasma concentration in the rf capacitive discharge registered with a Langmuir probe. An abrupt increase of electron temperature and glow intensity near the tube wall in the weak-current $\alpha$-mode of the rf capacitive discharge is revealed, the radial distribution of plasma concentration and ion flow to the electrodes possessing a maximum near the radial sheath boundary. In the $\gamma$-mode of the rf capacitive discharge the electron temperature decrease in the total plasma volume leads to an electric field weakening and the peak of the glow intensity near the tube wall vanishes. The radial sheath thickness in the $\alpha$-mode of the rf capacitive discharge obtained with $2 \mathrm{D}$ simulation experiences pulsations during the rf field period, the changing radial electric field heating electrons and increasing the plasma concentration near the boundary of the radial sheath.
\end{abstract}

(c) 2009 Elsevier Ltd. All rights reserved.

\section{Introduction}

The radio frequency (rf) capacitive gas discharge is widely used in various technological processes: plasma etching and modification of different materials [1-4], depositing nitride, oxide, diamond-like and other thin films [4-6], plasma cleaning of technological gas-discharge chambers [7], plasma chemistry [8], pumping gas lasers [9], sterilizing medical tools [10,11] etc. On performing, e.g., etching semiconductor plates and depositing films it is important to possess a uniform distribution of plasma parameters across the electrode (plate) area. Therefore the information on the radial structure of the rf discharge is of considerable interest.

However, in spite of the importance of this issue, only several theoretical papers were devoted to studying the radial structure of a symmetric rf discharge. The authors of papers [12-14] presented a two-dimensional self-consistent nonequilibrium fluid model for simulating rf glow discharges in chambers with a cylindrically symmetric design. They found that the strength of the radial electric field in a sheath near the tube wall exceeded the value of the axial electric field at the central region of the chamber considerably. This led to the appearance of a plasma concentration peak near the boundary of the radial sheath. Lieberman et al. [15] developed the electromagnetic theory for a parallel-plate discharge which accounted for the propagation of surface and evanescent waves

\footnotetext{
* Corresponding author. Tel.: +380 577111967.

E-mail address: lisovskiy@yahoo.com (V.A. Lisovskiy).
}

from the discharge edge to the centre and the role of capacitive and inductive fields in driving the power absorption. They studied the distribution of the capacitive, inductive and total power across the electrode area at different frequencies and plasma concentration values. Overzet and Hopkins [16] obtained with a Langmuir probe that in a GEC (Gaseous Electronics Conference) reference cell the radial profile of the plasma concentration possessed an abrupt peak near the electrode edge associated with an increased electric field in this region. Lai et al. [17] employed the SCCM $^{\mathrm{TM}}$ asymmetric discharge chamber $\left(\mathrm{SCCM}^{\mathrm{TM}}\right.$ is a registered trademark or a trademark of Tokyo Electron Limited in Japan and other countries) and obtained the donut-shaped profile of etching (with the smallest etching rate at the centre and at the very edge of the plate, whereas the largest etching rate was observed approximately at the distance of $5 / 8$ radius from the plate centre). They confirmed the nonuniform radial distribution of plasma parameters with probe measurements. Nonuniform radial distributions of plasma concentration in asymmetric chambers were also observed by Aoyagi et al. [18] (for oxygen at the frequency of $1 \mathrm{MHz}$ ) and by Takaki et al. [19] (for argon at the frequency of 13.56 MHz). Again Volynets et al. [20] observed singleand triple-peak distributions of optical emission of the rf discharge against the chamber diameter (for argon and fluorocarboncontaining gas mixtures at the frequency of $100 \mathrm{MHz}$ ).

As far as we know, the literature does not contain the experimental data on radial profiles of plasma parameters in a symmetric rf capacitive discharge. Therefore we registered with a Langmuir probe the radial profiles of electron temperature and plasma concentration in the rf discharge and took the photos of the glow intensity distribution of the discharge plasma across the electrode 
area. We also registered the radial distribution of the ion current density impinging the electrode surface. We obtained that in a weak-current $\alpha$-mode of the rf discharge the electron temperature and glow intensity possessed an abrupt peak near the tube wall, and the radial profiles of the plasma concentration and the ion flow to the electrodes approached their maxima near the boundary of the radial sheath. After the rf discharge transition to the strongcurrent $\gamma$-mode these peaks of electron temperature, glow intensity, plasma concentration and ion flow to the electrodes became weakly expressed or vanished. The maximum of plasma concentration in the $\gamma$-mode was located near the chamber axis, and the electron temperature became actually constant over the discharge cross section. With 2D simulation we demonstrated that the thickness of the radial sheath during the rf field period experienced pulsations heating electrons and increasing the plasma concentration near the boundary of the radial sheath in the $\alpha$-mode of the rf discharge. In the $\gamma$-mode this effect was weakened due to low electron temperature throughout the discharge volume.

\section{Experimental conditions}

\subsection{Plasma conditions and devices}

The experiments were performed in nitrogen and argon with the pressure values within the range $p=0.1-2.5$ Torr, the amplitude values of the rf voltage $U_{\mathrm{rf}} \leq 600 \mathrm{~V}$ and the rf field frequency $f=13.56 \mathrm{MHz}$. We performed measurements in the discharge chamber of $0.1 \mathrm{~m}$ in diameter with a gap between flat parallel stainless-steel electrodes of $L=0.022 \mathrm{~m}$ (Fig. 1). We also made a number of experiments with the chamber of $0.055 \mathrm{~m}$ in diameter and the gap between flat parallel stainless-steel electrodes of $0.04 \mathrm{~m}$. The rf potential was applied to one of the electrodes whereas another one was grounded.

Fig. 1 shows our experimental device. The fused silica tube was vacuum-sealed between the electrodes. The gas supply system fed gas through a multitude of tiny orifices in the grounded electrode. The discharge vessel was evacuated through a set of orifices in the same electrode. This design permitted to feed and pump out the gas uniformly across the electrode area what may be important in technological processes. For registering gas pressure within the range from $10^{-3}$ Torr to atmospheric one we employed the thermoelectric vacuum gauge. The flow rate of used gases was equal to $5 \mathrm{sccm}$ (standard cubic $\mathrm{cm}$ per minute). Gas was pumped out with the preliminary vacuum and turbo molecular pumps providing the

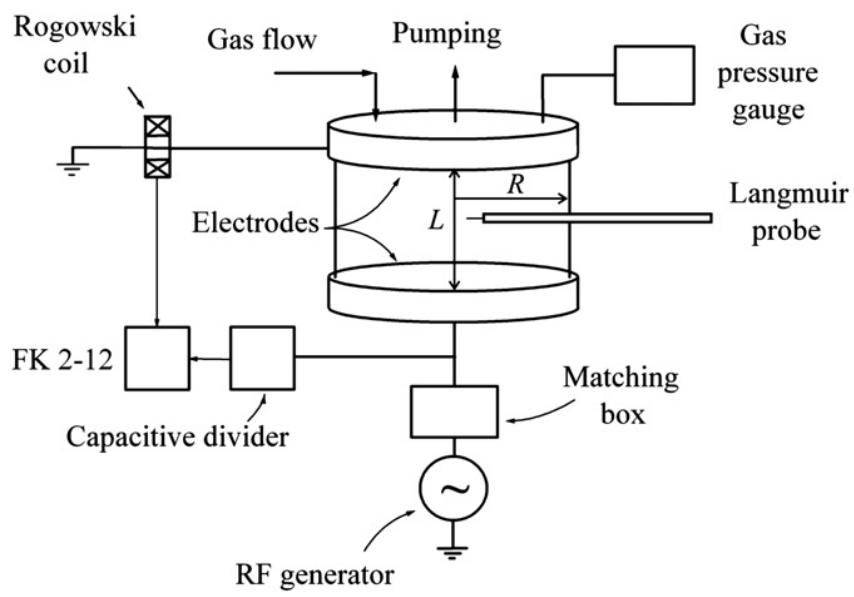

Fig. 1. Scheme of the experimental device (argon $-L=0.022 \mathrm{~m}, R=0.05 \mathrm{~m}$, nitrogen $\left.L=0.04 \mathrm{~m}, R=27.5 \times 10^{-3} \mathrm{~m}\right)$. limiting pressure order of $10^{-6}$ Torr. Rf generator was connected to the potential electrode via a matching box of П-type.

Rogowski coil was employed to determine the rf current amplitude, and its signal was fed to the FK 2-12 phase difference meter. A signal from the capacitive divider connected to the $\mathrm{rf}$ electrode was fed to another input of FK 2-12. This gave an opportunity to measure the amplitudes of rf current, rf voltage and the phase shift angle $\varphi$ between them. The FK 2-12 phase difference meter is capable of registering the amplitudes of alternating signals within the frequency range $f_{1}=1 \mathrm{MHz}$ to $1 \mathrm{GHz}$ and the phase shift $\varphi$ between the signals (between the rf voltage and rf current in our case).

In order to register the radial distribution of the ion current we designed an electrode (of $0.055 \mathrm{~m}$ in diameter) with 9 current collectors located in a straight line along the electrode diameter. The diameter of each collector was $0.003 \mathrm{~m}$. The collectors were insulated electrically from the electrode, and the collecting surfaces of them and the electrode surface were in the same plane. The inter-electrode gap of this chamber was $L=0.04 \mathrm{~m}$. Fig. 2 shows the discharge chamber equipped with such an electrode. The collected current flew through the resistors of $1 \mathrm{k} \Omega$, and the voltage drop across each resistor was fed to PC. The resistors were supplied with capacitors connected in parallel with them to remove the effect of rf current on ion current registration. Currents through all collectors were registered simultaneously, the measurements were repeated

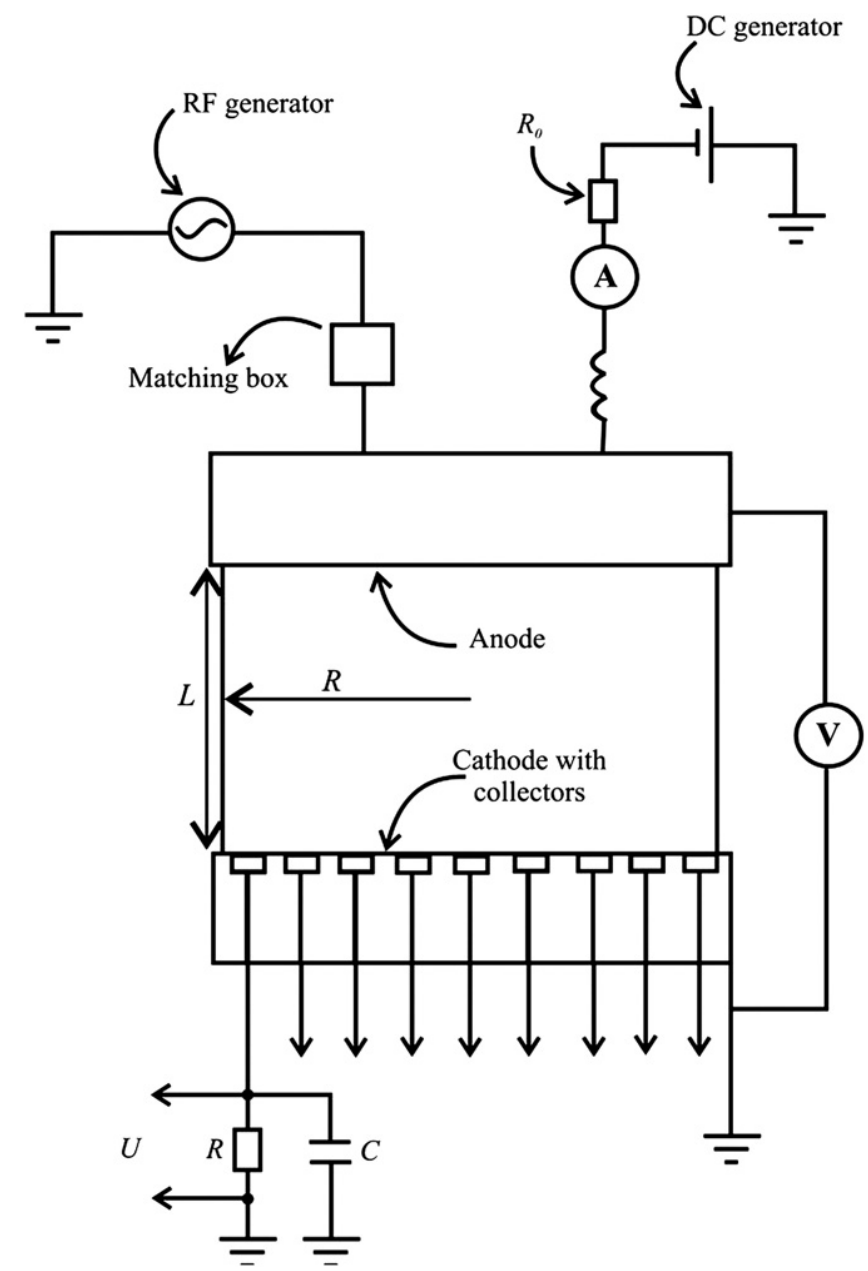

Fig. 2. Scheme of the experimental device having an electrode with built-in collectors, $L=0.04 \mathrm{~m}, R=27.5 \times 10^{-3} \mathrm{~m}$. 


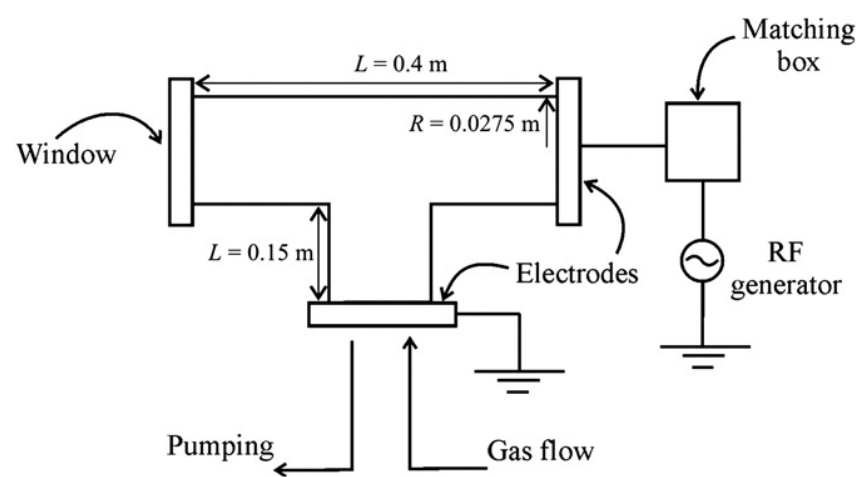

Fig. 3. Scheme of the experimental device with the T-shaped tube, $R=27.5 \times 10^{-3} \mathrm{~m}$.

20 times and the data obtained were averaged. In the dc discharge the current collected by the cathode consists almost of the current of positive ions. In the rf discharge the electrodes collect ions during the total period of the rf field, but electrons also come to the electrode (in the form of pulses in the "anode" phase of the nearelectrode sheath). Therefore we applied a small dc potential $(20 \mathrm{~V})$ to the electrode with collectors. It did not affect the rf discharge characteristics essentially but permitted to register just the ion current density coming to the electrode.

Experiments were also carried out in a T-shaped tube (see Fig. 3), the rf potential being applied to the electrode at one end of the horizontal part of T, whereas another electrode (at the bottom of T) was grounded. Photos were taken through a window at the opposite end of the horizontal part of T exposing the rf electrode and the images were digitized with MathCad 2001 Pro. The length of the horizontal section of $\mathrm{T}$ (the distance between the rf electrode and the window) was equal to $0.4 \mathrm{~m}$. The length from anode to the horizontal part of $\mathrm{T}$ was equal to $0.15 \mathrm{~m}$. Photos of the discharge were made with the Canon IXUS 500 digital camera.

\subsection{Langmuir probe measurements description}

Electron temperature $T_{\mathrm{e}}$, plasma potential and plasma concentration $n_{\mathrm{i}}$ were established from the measurements with a single cylindrical nichrome Langmuir probe (probe length was $5.5 \times 10^{-3} \mathrm{~m}$, probe diameter was $\left.0.18 \times 10^{-3} \mathrm{~m}\right)$, which might move along the tube radius in the central plane. Fig. 4a shows the example of the obtained probe CVC (current-voltage characteristic). We employed the following technique to determine the electron temperature. The ion branch of the current-voltage characteristics matches a straight line with good accuracy. Therefore we first chose the approximation formula describing the ion branch $I_{\mathrm{p}, \mathrm{i}}$. Then we subtracted the ion current from the registered probe current $I_{\mathrm{p}}$ and determined the electron current $I_{\mathrm{e}}$ to the probe, namely, $I_{\mathrm{e}}=I_{\mathrm{p}}-I_{\mathrm{p}, \mathrm{i}}$. The dependence of the natural logarithm of the electron current $\ln \left(I_{\mathrm{e}}\right)$ on the probe potential $U_{\mathrm{p}}$ (see Fig. $4 \mathrm{~b}$ ) within the range between the floating and plasma potentials possesses a linear section (under the condition that cold electrons possess a Maxwellian distribution with the temperature $T_{\mathrm{e}}$ ). Then the electron temperature is determined from the angle at which this linear section is tilted according to the formula $T_{\mathrm{e}}=\Delta U_{\mathrm{p}} / \Delta \ln \left(I_{\mathrm{e}}\right)[\mathrm{eV}]$, which is determined by the increments of the electron current logarithm $\Delta \ln \left(I_{\mathrm{e}}\right)$ and the probe $\Delta U_{\mathrm{p}}$, corresponding to the linear section in question.

Differentiating the registered electron current $I_{\mathrm{e}}$ twice with respect to $U_{\mathrm{p}}$ we can determine the electron energy probability function (EEPF) $f_{\mathrm{e}}(v(V))(v$ is the electron velocity, $V$ is the retarding potential for the electron current, counted from the plasma

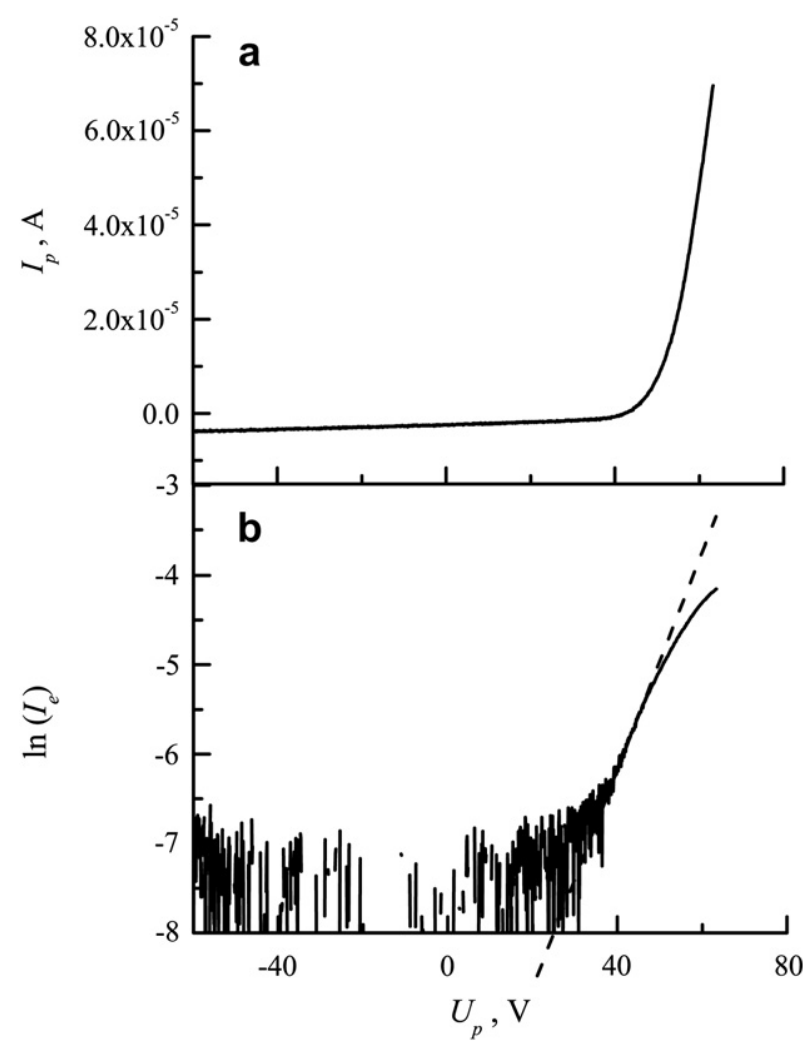

Fig. 4. a) Example of the probe CVC, nitrogen, $p=0.5$ Torr, $U_{\mathrm{rf}}=133 \mathrm{~V}$, b) Dependence of $\ln \left(I_{\mathrm{e}}\right)$ on probe potential.

potential $\varphi_{\mathrm{pl}}$ and determined according to the formula $\left.V=\varphi_{\mathrm{pl}}-U_{\mathrm{p}}\right)$, using formula (6.6.14) [4]

$\frac{d^{2} I_{\mathrm{e}}}{d U_{\mathrm{p}}^{2}}=\frac{2 \pi e^{3}}{m^{2}} A \cdot f_{e}(v(V))$.

In this formula $e$ and $m$ are electron charge and mass, respectively, $A$ is the probe collecting area.

The rf current penetration in the probe circuit may distort the CVC of the probe immersed in the rf discharge plasma. The plasma potential oscillates, and the current collected with the probe also experiences time variations due to rf oscillations of the voltage drop across the probe sheath. Besides the floating potential of the probe decreases, and the electron temperature we got from the probe CVC is larger and the plasma concentration is smaller than the actual values. Therefore an additional (auxiliary) compensation electrode is introduced [21], which is connected to the measuring probe via a capacitor. The compensation electrode increases the capacitance and decreases the resistance of the near-probe sheath [22]. A compensation (auxiliary) electrode was located in the same plane with the probe (its area was 70 times larger than the Langmuir probe area). The probe circuit also contained the resonance filter tuned to the frequency of $13.56 \mathrm{MHz}$ as well as the choke of $109 \mathrm{mH}$ inductance permitting to prevent the rf current from penetrating the probe measurement circuit. Our probe measurement circuit was similar to one suggested in paper [10], therefore it is hardly expedient to describe it here. Moving the probe along the tube axis we registered the axial distribution of plasma parameters.

Plasma concentration $n_{\mathrm{i}}$ was calculated from the ion branch of the probe current $I_{\mathrm{p}, \mathrm{i}}$ and the electron temperature $T_{\mathrm{e}}$ determined according to the technique described in papers $[23,24]$. To this end we employed the formula 
$I_{p, i}=I_{i}^{*} \cdot I^{*}$,

where

$I^{*}=A \sqrt{\frac{k T_{\mathrm{e}}}{2 \pi M_{i}}} n_{i} e$,

$I_{i}^{*}=\gamma_{1} \gamma_{2} I_{L}^{*}$,

$k$ is the Boltzmann constant, $M_{\mathrm{i}}$ is the ion mass; $I_{\mathrm{L}}^{*}$ is the Laframboise current

$I_{L}^{*}=\frac{2}{\sqrt{\pi}} \sqrt{\eta}$,

$\gamma_{1}=1+\left(\frac{I_{A}^{*}}{I_{L}^{*}}-1\right) \cdot X_{i} \quad X_{i}<1$,

$\gamma_{1}=\frac{I_{A}^{*}}{I_{L}^{*}} \quad X_{i} \geq 1$

$\gamma_{2}=\frac{3-2 \cdot \exp \left(-X_{i}\right)}{1+2 \cdot X_{i}} \quad X_{i}<1$

$\gamma_{2}=\frac{3-2 \cdot \exp \left(-X_{i}\right)}{2 \cdot\left(1+X_{i}\right)} \quad X_{i} \geq 1$.

Here

$X_{i}=\frac{\sqrt{\bar{\eta}}}{D_{\lambda} K_{i}}$

$\bar{\eta}=m(\eta+3.5)-4, \quad m \approx 0.59+1.86\left(D_{\lambda}\right)^{0.47}, \quad \eta=e U_{\mathrm{p}} / k T_{\mathrm{e}}$ is the dimensionless probe current vanishing at the plasma potential, $K_{\mathrm{i}}=\lambda_{\mathrm{i}} / R$ ( $R$ is the probe radius, $\lambda_{\mathrm{i}}$ is the ion mean free path inversely proportional to gas pressure), $D_{\lambda}=R / \lambda_{\mathrm{D}}$ ( $\lambda_{\mathrm{D}}$ is the Debye length depending on plasma concentration and electron temperature), $I_{A}^{*}=0.5(\eta / 0.17)^{\alpha}, \alpha=0.65\left(D_{\lambda}\right)^{0.47}$. Thus, the coefficients $\gamma_{1}$ and $\gamma_{2}$ are functions of the ion concentration, electron temperature and gas pressure $[23,24]$ :

$\gamma_{1}, \gamma_{2}=f_{1,2}\left(n_{i}, T_{\mathrm{e}}, p\right)$,

Inserting (3)-(7) into (2) furnishes the equation the left hand part of which contains the ion current we registered with the probe and the right hand part of which is the function of the gas pressure, electron temperature and plasma concentration. Solving this equation we get the concentration of positive ions. In so doing we use the values of the electron temperature determined from the probe CVCs (employing the linear section of the graph of the electron current to the probe constructed to the semi-logarithmic scale).

\section{Experimental results}

Fig. 5 shows the radial profiles of the electron temperature for two values of the argon pressure and various rf voltage values registered in the central plane of the discharge. It is clear from the figure that in the weak-current $\alpha$-mode [4,9,25-35] of the rf discharge (the curve for $U_{\mathrm{rf}}=50 \mathrm{~V}, p=0.1$ Torr and the curves for $U_{\mathrm{rf}}=100$ and $165 \mathrm{~V}$, $p=1$ Torr) counting from the discharge axis, the electron temperature first remains constant or increases weakly. However on approaching the wall of the discharge tube the electron temperature $T_{\mathrm{e}}$ increases abruptly, its maximum value exceeding $T_{\mathrm{e}}$ near the discharge axis almost more than twice. On increasing the rf voltage the electron temperature in the $\alpha$-mode decreases weakly in the total discharge volume, the peak of the electron temperature near the tube wall still remaining. But under the $\alpha-\gamma$ transition of the rf discharge the magnitude of the electron temperature peak near the tube wall
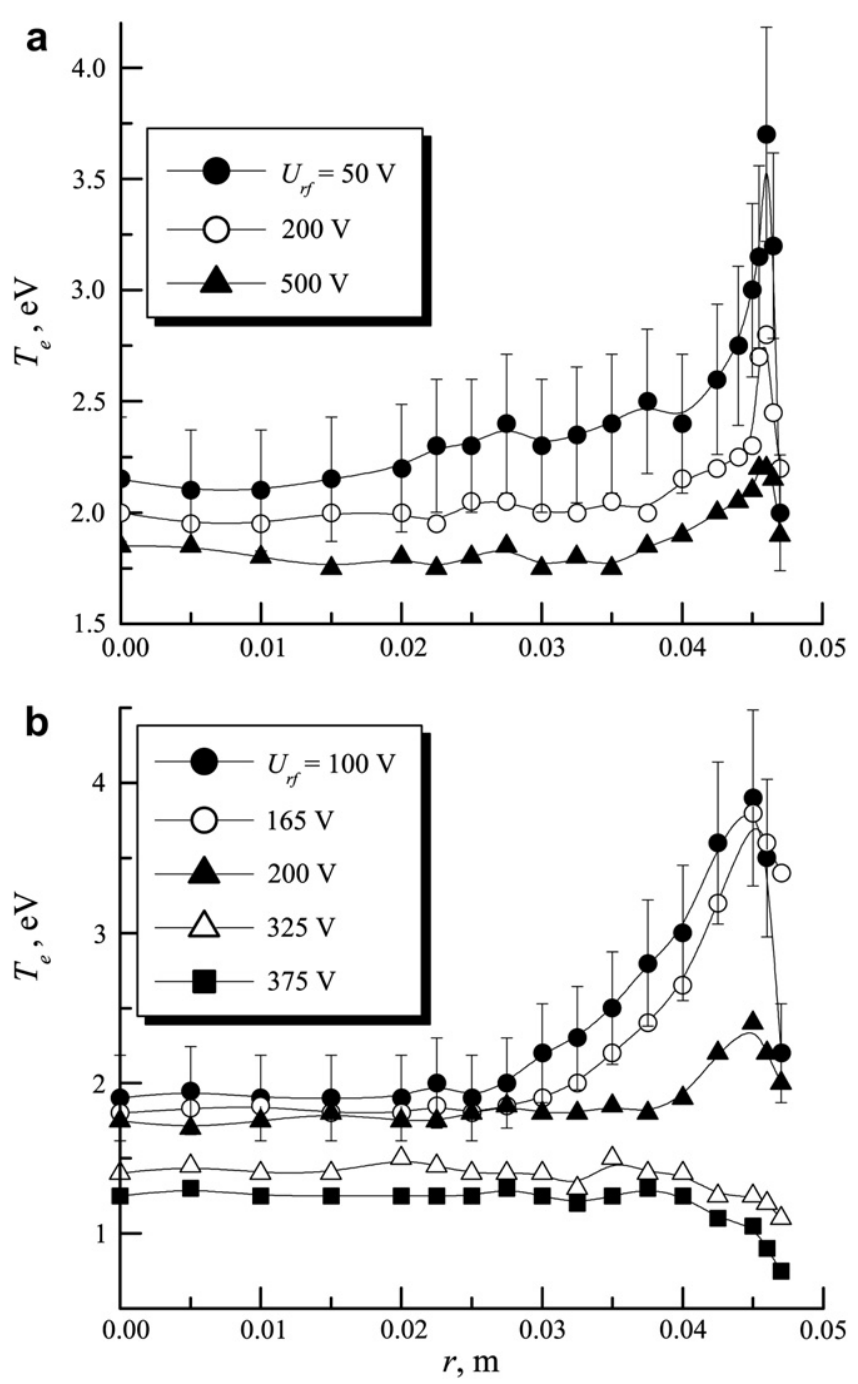

Fig. 5. Radial profiles of electron temperature with argon pressure of 0.1 Torr (a) and 1 Torr (b) and various RF voltage values. The inter-electrode gap is $0.022 \mathrm{~m}$, the chamber diameter is $0.1 \mathrm{~m}$.

decreases abruptly (the curve for $U_{\mathrm{rf}}=200 \mathrm{~V}, p=1$ Torr), and then in the strong-current $\gamma$-mode this peak of electron temperature is absent.

We observed a similar behavior of the electron temperature in Fig. 6 for the rf discharge in nitrogen in the chamber of $0.055 \mathrm{~m}$ in diameter and the inter-electrode gap of $0.04 \mathrm{~m}$. Electron temperature remained approximately constant from the chamber axis to $r \approx 0.022 \mathrm{~m}$, then we observed an abrupt $T_{\mathrm{e}}$ increase near the tube wall exceeding approximately twice the value in the central region of the discharge. After the rf discharge transition from $\alpha$ - to $\gamma$-mode the peak of the electron temperature near the tube wall experienced a fast decrease and then actually vanished (see the curve for $U_{\mathrm{rf}}=333 \mathrm{~V}$ ). The plasma concentration in the $\alpha$-mode increased slowly when the probe moved from the central region to the tube wall approaching a maximum and then decreasing fast in the radial sheath (near the tube wall). For all rf voltage values we studied the radial profiles of the plasma concentration happened to be far from the profile given by the Bessel function of zero order $J_{0}(2.405 r / R)$ shown in Fig. 6 with a broken line. The profiles also differed from the uniform distribution of the plasma concentration assumed in onedimensional models of the rf discharge. Electrons whose energy is acquired in the rf electric field in the plasma volume due to Joule heating perform a portion of ionizing collisions in the quasi-neutral plasma. The ionization by these electrons together with the 


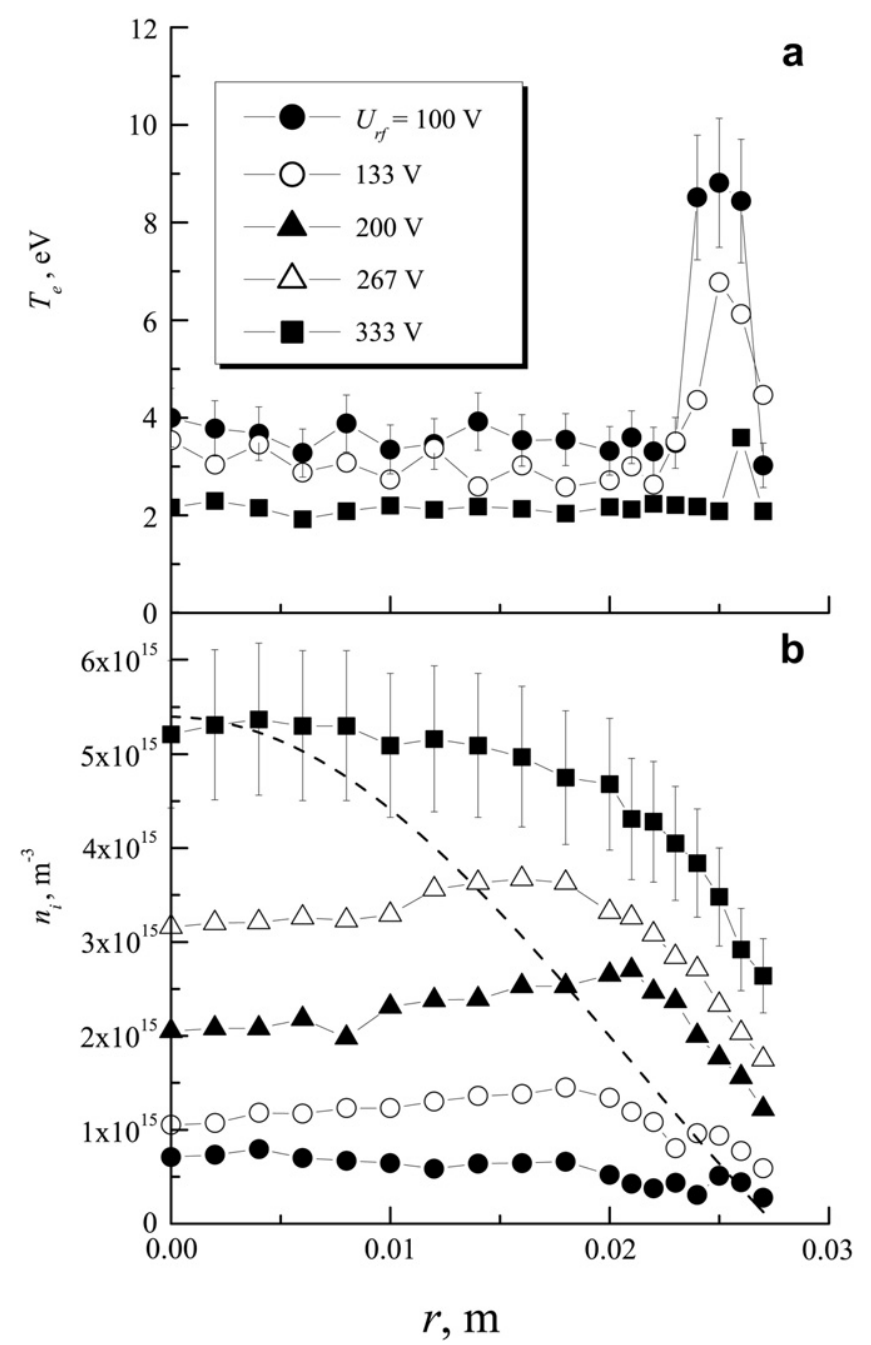

Fig. 6. Radial profiles of electron temperature (a) and plasma concentration (b) at nitrogen pressure of 0.5 Torr. The inter-electrode gap is $40 \times 10^{-3} \mathrm{~m}$, the chamber diameter is $0.055 \mathrm{~m}$. The broken line shows the plasma concentration profile described by Bessel function of zero order $J_{0}(2.405 r / R)$.

diffusion escape of charged particles to the tube walls would lead to the radial profile of the plasma concentration described by the Bessel function of zero order $J_{0}(2.405 r / R)$. However, as we already said above, the considerable energy is gained by the electrons swept out of the near-electrode sheath in the cathode phase (when the oscillating sheath boundary is moving off the electrode) [27]. At low gas pressure these electrons may perform ionizing collisions in the plasma volume increasing the concentration of charged particles. In the $\gamma$-mode the electron avalanches develop in the near-electrode sheaths, and a flow of fast electrons is moving from the sheaths into the plasma performing an intense ionization of gas molecules. As the sheaths are the sources of fast electrons, this flow has to be uniformly distributed over the discharge cross section. Probably, due to this reason the radial profile of plasma concentration we measured contains a larger electron concentration far from the discharge axis compared with Bessel's profile. Besides, as will be shown below, a considerable contribution to gas molecule ionization near tube walls is made by electrons accelerated in the oscillating radial sheath.

One can argue on the validity of Langmuir probes in spacecharge regions, in presence of anisotropic velocity distributions, etc. but even when the probe does not provide high-accuracy data, it allows at least to detect some basic features.
Figs. 5 and 6 allow us to draw a conclusion that the enhanced electron heating near the tube wall is observed in $\alpha$-mode of the rf capacitive discharge in noble as well as in molecular gases. Therefore the mechanism of such a heating is perhaps identical in various gases. An abrupt increase of electron temperature near the tube wall has to furnish a more intense glow in this region. To check this and study the radial structure of discharge glow we designed a T-shaped tube with a window shown in Fig. 3. Fig. 7a presents the photo of the discharge glow made through the window of the Ttube. We see a bright pink glow extending from the boundary of the near-electrode sheath to the window at the butt of the horizontal section of the T-shaped tube. Near the tube wall (at the photo's
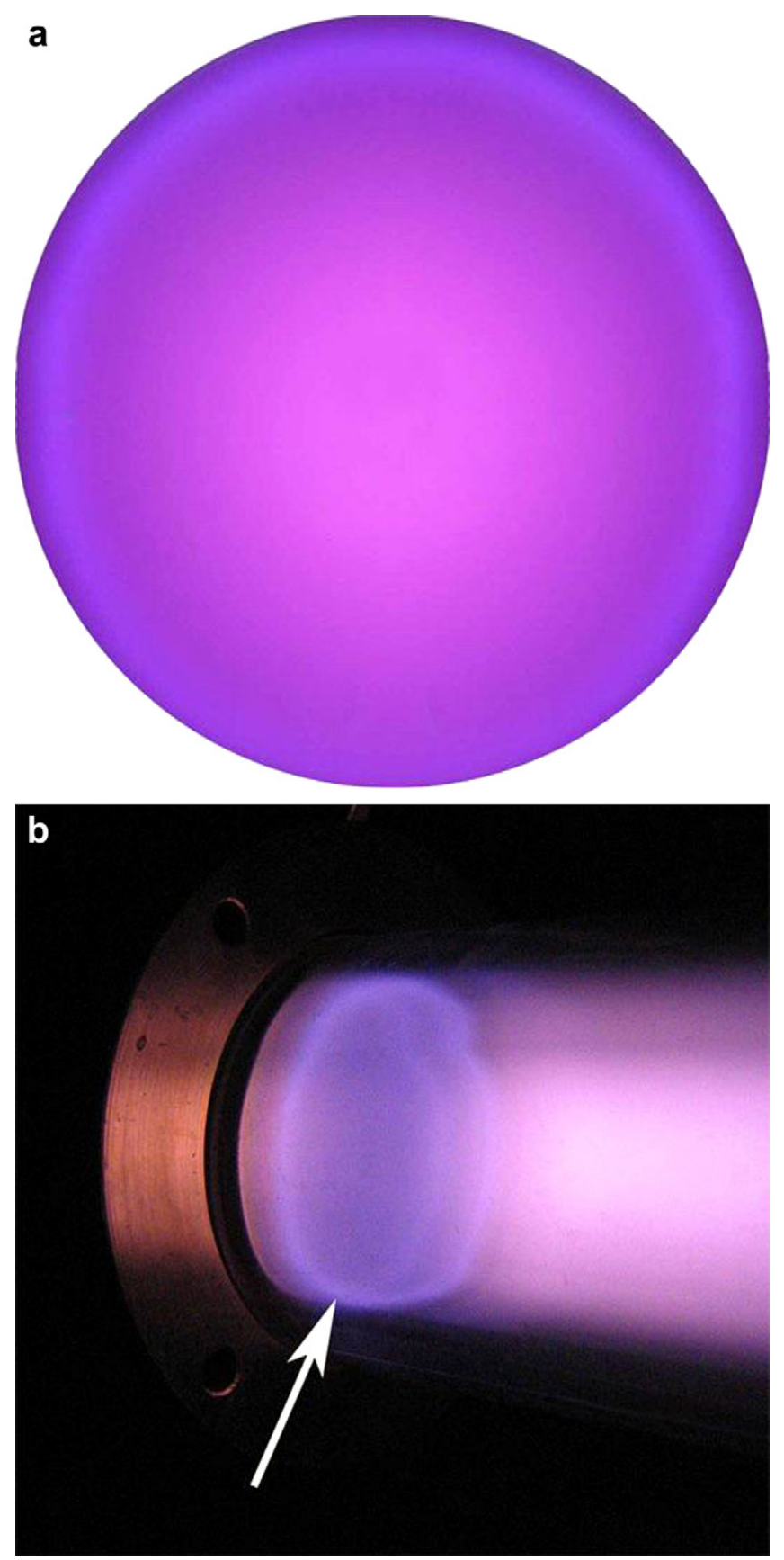

Fig. 7. a) Photo of discharge glow, b) the arrow indicates the region of enhanced electron heating near the wall of the discharge tube. T-shaped tube, nitrogen, $p=0.5$ Torr, $U_{\mathrm{rf}}=367 \mathrm{~V}$. 
edge) we observe a blue glow produced in the region where the near-electrode sheath contacts the tube wall.

Fig. 8 depicts the radial profiles of the discharge glow intensity for three values of nitrogen pressure obtained with the T-shaped tube. To this end we took a series of photos similar to one presented in Fig. 7a (regretfully we had no opportunity to register the axial or radial distributions of intensities of separate lines of discharge radiation). As the discharge glow has a blue colour near the tube wall, we presented in Fig. 8 the profiles of the discharge glow in the blue part of the spectrum (in order to get a better image of the glow peak near the tube wall). At the lowest value of the discharge sustainment voltage at all values of nitrogen pressure we studied the maximum of the blue glow near the tube wall was not observed, and it appeared only with the rf voltage growing. Under these conditions the radial sheath was thick and dark because of the low plasma concentration in the discharge. Increasing the rf voltage involved the growth of plasma concentration, the discharge glow occupied the total electrode surface, and then near the tube wall a blue glow appeared having the shape of a ring and well observed with a naked eye. At low nitrogen pressure ( $p=0.1$ Torr, Fig. $8 a$ ) the width of the
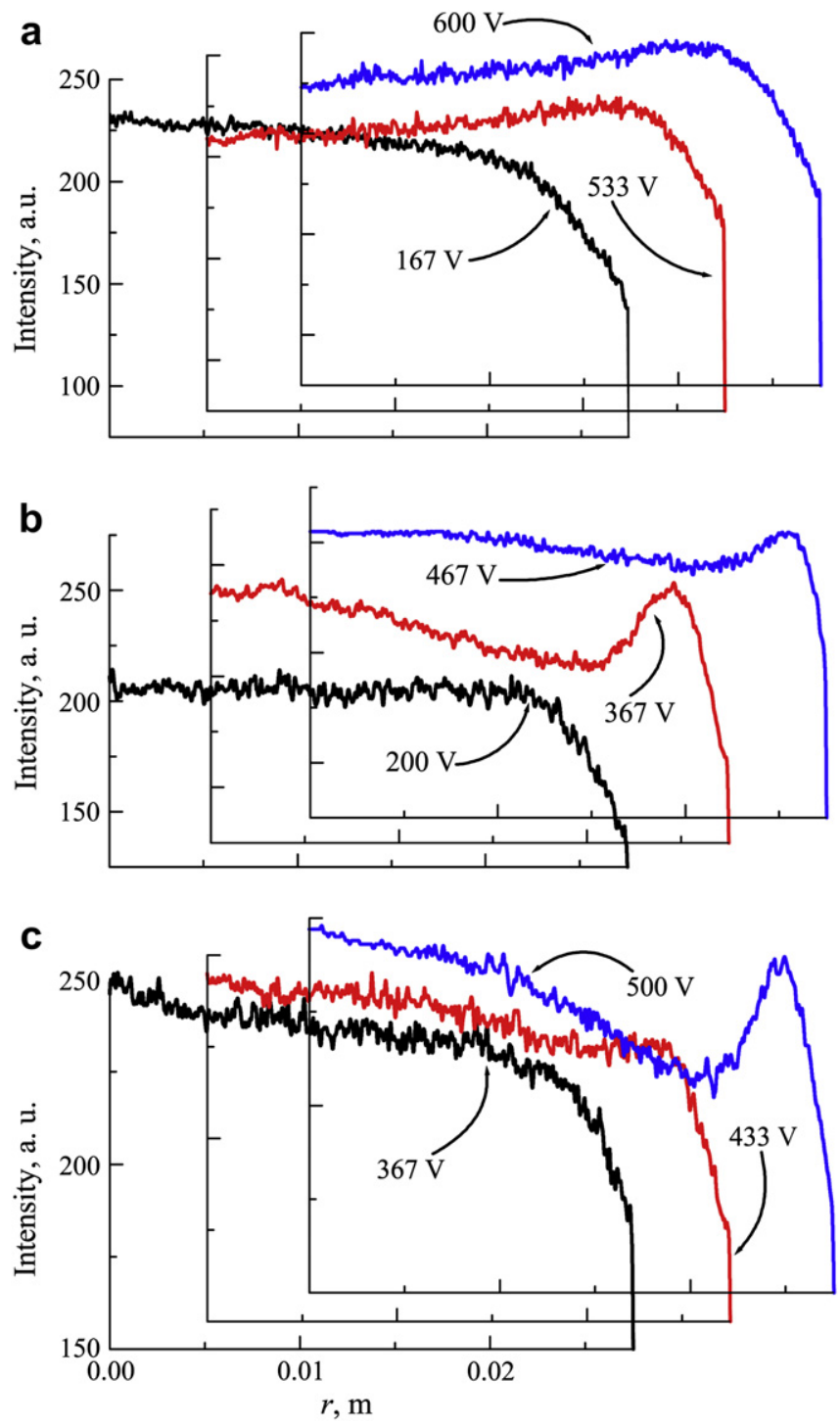

Fig. 8. Radial profiles of discharge glow intensity at nitrogen pressure of 0.1 Torr (a), 0.5 Torr (b) and 1 Torr (c). T-shaped tube. blue ring was not less than $0.01 \mathrm{~m}$, the intensity of its glow grew with the $\mathrm{rf}$ voltage increasing. At the nitrogen pressure of $p=0.5$ Torr (Fig. $8 \mathrm{~b}$ ) in the $\alpha$-mode with the rf voltage increasing a blue ring appeared first to have around $0.005 \mathrm{~m}$ in width, and its intensity grew. A further increase of the rf voltage led to the discharge transition from $\alpha$ - to $\gamma$-mode, the blue ring became narrower and it was located closer to the tube wall, and after the complete transition of the rf discharge to $\gamma$-mode the blue ring disappeared. We observed a similar behaviour of the discharge glow at the nitrogen pressure $p=1$ Torr (Fig. 8c). At this pressure value we did not manage to get the transition from $\alpha$-to $\gamma$-mode because of the ignition of a parasitic discharge in the orifices of the grounded electrode, through which the gas was pumped out of the chamber, and we did not present the data for $\gamma$-mode in Fig. 8c.

Fig. 9 shows the axial profiles of $T_{\mathrm{e}}$ in argon for different $\mathrm{rf}$ voltage values. These profiles possess maxima near the axial boundaries of near-electrode sheaths in $\alpha$-mode of the rf discharge, but after the transition of the discharge to the $\gamma$-mode these peaks disappear.

In contrast to the positive column of the dc discharge where the average energy of electrons decreases from the tube centre to the wall [36], a reverse situation is observed in rf discharge. Fig. 10 shows the electron energy probability functions (EEPF) for the rf discharge in argon in $\alpha$-mode registered in the central plane. The figure demonstrates that at the rf discharge centre the EEPF possesses a Maxwellian group of cold electrons and a small "tail" of high energy electrons. Counting from the discharge centre the electron concentration in the EEPF Maxwellian group grows; the energy and number of electrons in the EEPF "tail" are also increased. The maximum number of high energy electrons is observed near the radial sheath boundary $(r=0.04 \mathrm{~m}$ in Fig. 10). Near the tube wall itself the concentrations of electrons in the Maxwellian group as well as in the EEPF "tail" decrease substantially.

The profiles of the ion current density to the electrode may serve an additional argument in favour of the radial dependence of plasma parameters we studied. In this experiment we employed the grounded electrode with 9 in-built collectors located along the total diameter of the electrode. Fig. 11 presents the registered data. The figure shows that in $\alpha$-mode the current density maxima the electrode collects are located not near the chamber axis but they are shifted to the tube radial wall. Obviously this radial profile of

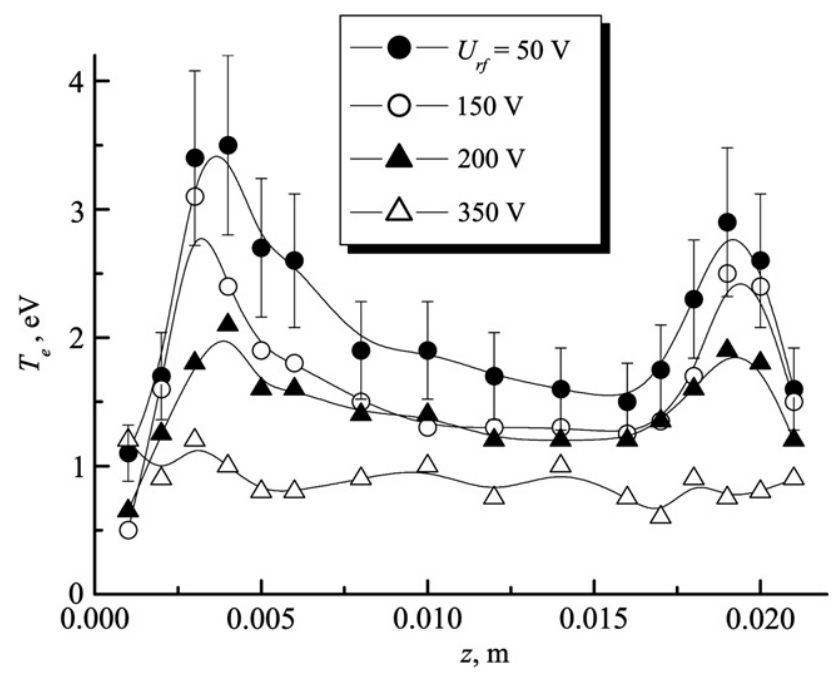

Fig. 9. Axial profiles of electron temperature at argon pressure of 1 Torr and different rf voltage values. The inter-electrode gap is $L=0.022 \mathrm{~m}$, the chamber diameter is $d=0.1 \mathrm{~m}$. 


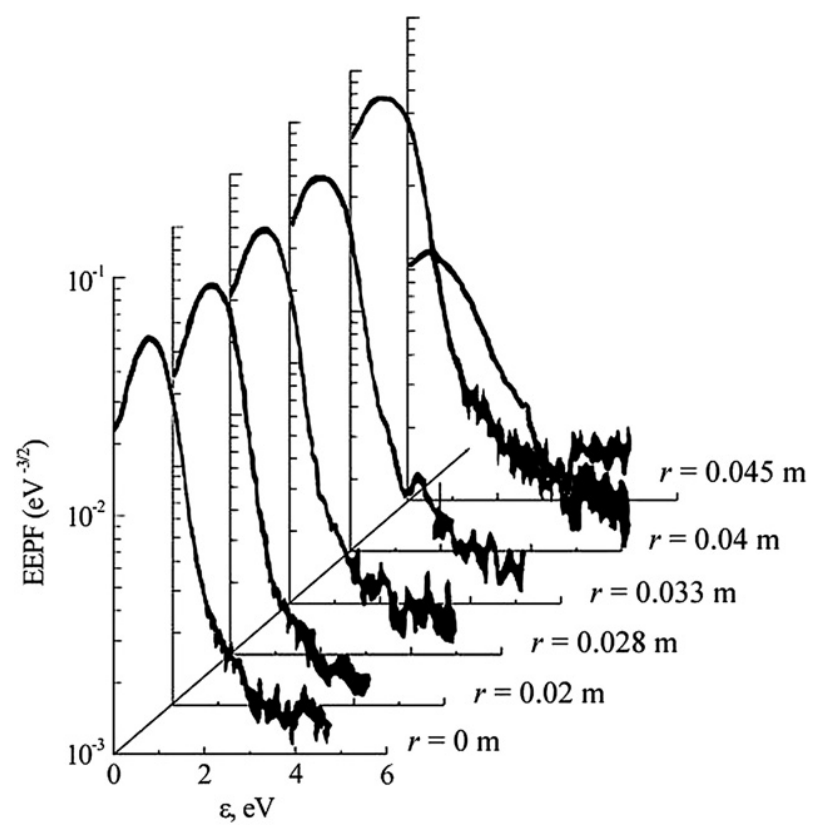

Fig. 10. Electron energy probability functions for $z=11 \mathrm{~mm}$ and different distances $r$ from the chamber axis. Argon, $p=1$ Torr, $U_{\mathrm{rf}}=175 \mathrm{~V}, L=0.022 \mathrm{~m}$.

current density has to be in agreement with the plasma concentration profile at the near-electrode sheath boundary. After the discharge transition to $\gamma$-mode the current density distribution becomes more uniform over the electrode surface (Fig. 11a). However we observe the appearance of maxima in the current density radial profile near the chamber axis at large rf voltage values and especially at higher pressure values (Fig. 11b).
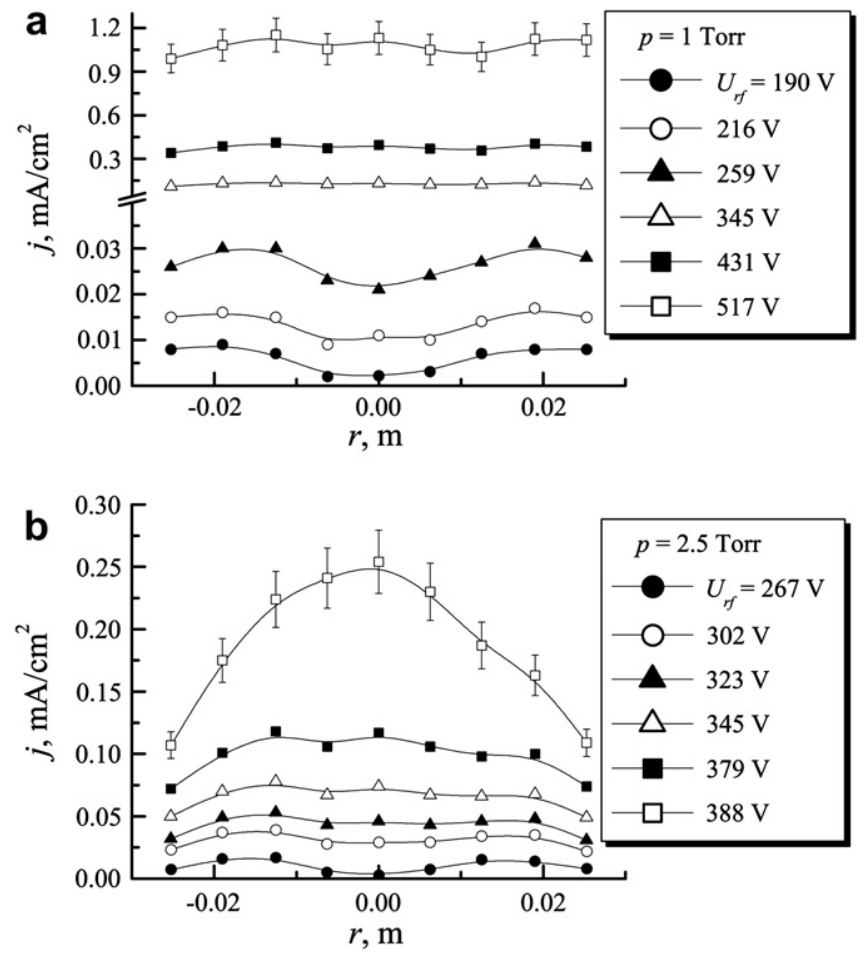

Fig. 11. Radial profiles of ion current density at the electrode at nitrogen pressure 1 Torr (a) and 2.5 Torr (b) and different rf voltage values, $L=40 \times 10^{-3} \mathrm{~m}$, $R=27.5 \times 10^{-3} \mathrm{~m}$.

\section{Discussion}

As is known [4,9,25-35], a rf capacitive discharge may burn in two different modes: a weak-current $(\alpha-)$ and strong-current $(\gamma$ mode). In the $\alpha$-mode the electrons acquire their energy for ionizing gas atoms in the rf field in the quasi-neutral plasma, the electron emission from the surface of the electrodes not playing a substantial role in the discharge sustainment. The conductance of near-electrode sheaths is small, and the current between the plasma and the electrodes is mainly due to the displacement current. However the authors of paper [27] employed a hydrodynamic modeling and demonstrated that in the $\alpha$-mode the electrons filled the near-electrode sheath during its anode phase. In the cathode phase the moving boundary of the expanding sheath sweeps these electrons back into the plasma, and they may gain energy due to an increased electric field in the sheath. The modeling [27] also demonstrated that the ionization rate of gas molecules via electrons swept by the expanding sheath boundary exceeded the ionization rate by electrons obtaining their energy from the rf electric field in the plasma volume. In the $\gamma$-mode electron avalanches develop in the near-electrode sheaths, the ionization of gas atoms via electron impact occurs mainly near the sheath boundary and electron emission from the electrodes affects the electron multiplication and discharge sustainment substantially. Near-electrode sheaths in the $\gamma$-mode possess a substantial conductance and they are similar to a cathode sheath of the dc glow discharge in their characteristics.

In this work we aimed to study the radial structure of the rf capacitive discharge. As was said above, papers [12-14] had shown via simulation that an increased heating of electrons had to be observed near the dielectric radial wall of the discharge tube. But, to our knowledge, the literature does not contain experimental radial profiles of plasma parameters of the symmetrical rf discharge. Therefore we performed a number of measurements with a Langmuir probe, a discharge chamber with collectors to measure radial profiles of the current density and we also employed a T-shaped tube to get the photos of the discharge glow in the $\alpha$-as well as in the $\gamma$-mode and digitized them.

Now let us consider the mechanism of enhanced heating of electrons near the tube wall. In the plasma of sufficiently high concentration $\left(n_{\mathrm{e}}>10^{14} \mathrm{~m}^{-3}\right)$ charged particles move out of the discharge volume to tube walls due to ambipolar diffusion [35]. The ambipolar electric field makes equal the flows of electrons and ions leaving the plasma region (and falling on the tube wall in this case). As a result the tube wall acquires a floating potential $\varphi_{\mathrm{pl}}$ which is negative with respect to the plasma. Thus a potential barrier is formed near the wall decelerating the electrons incident on it. Only the fastest electrons possessing the energy $\varepsilon \geq e \varphi_{\mathrm{pl}}$ may approach the tube wall. As was shown in experiment [36], in the positive column of the dc discharge the average energy of electrons moving in the ambipolar field from the discharge centre to the tube wall decreases. Slow electrons do not approach the wall and they return back to the plasma accelerating in the ambipolar field [37] and forming a weak peak of electron temperature near the radial boundary of the sheath. The strength of the ambipolar field $E_{\mathrm{r}}$ is equal to:

$E_{r} \approx-\frac{D_{e}}{\mu_{e}} \cdot \frac{1}{n} \cdot \nabla n=-\frac{k T_{\mathrm{e}}}{e} \cdot \frac{1}{n} \cdot \nabla n$,

where $\mu_{e}$ is electron mobility, $D_{\mathrm{e}}$ is the coefficient of electron free diffusion [35]. Thus the magnitude of the ambipolar electric field $E_{\mathrm{r}}$ is determined by the electron temperature $T_{\mathrm{e}}$ in the plasma. We may expect that the ambipolar electric field $E_{\mathrm{r}}$ will be at maximum in the regions of enhanced electron heating. 
Axial profiles of $T_{\mathrm{e}}$ we registered in argon at different rf voltage values (see Fig. 9), possess their maxima near the axial boundaries of near-electrode sheaths in $\alpha$-mode of the rf discharge (the curves for $U_{\mathrm{rf}}=50 \mathrm{~V}$ and $150 \mathrm{~V}$ ). As we said above, in $\alpha$-mode the largest heating of electrons occurs in the cathode phase of near-electrode sheaths when the electrons penetrating the sheaths in the anode phase are swept back into the plasma when the oscillating boundary is moving from the electrode [27] gaining a substantial energy. Under the transition from $\alpha$ - to $\gamma$-mode the electron avalanches develop in the sheaths, and a flow (beam) of high energy electrons is injected from the near-electrode sheaths into the plasma. A restructuring of the rf discharge occurs, and the characteristics of near-electrode sheaths become similar to those of the cathode sheath of the dc discharge [25]. The regions of plasma adjacent to near-electrode sheaths become similar to a negative glow due to the presence of a flow of high energy electrons (at sufficiently high gas pressure and a long gap between the electrodes the Faraday dark spaces also appear). In these regions of the dc discharge, as is known [4,35], the electron temperature is small, because the flow of high energy electrons carries a considerable portion of the discharge current and produces an intense ionization of gas molecules, and there is no necessity to sustain the ionization level in these regions with plasma electrons. Therefore in the $\gamma$ mode the maxima of the axial profiles of electron temperature near the electrode boundaries almost vanish.

Electron heating in the rf discharge near its radial wall may be associated with the processes inside the near-electrode sheath, and it might be most clearly pronounced in short discharge tubes (several centimetres long). Fig. $7 \mathrm{~b}$ presents the photo of the rf discharge (burning in the T-shaped tube) where we can observe that the glow is the brightest just in the region of contact between the near-electrode sheath and the tube wall. However such a heating may also be present in long discharge tubes, because the dielectric walls of a discharge tube acquire a negative charge and a considerable radial field is established. A superposition of this radial field and a longitudinal rf electric field would lead to an enhanced heating of electrons just near the tube wall.

\section{Simulation results}

For better understanding the processes occurring in a symmetric rf discharge we performed a simulation with the OOPIC Pro code (Tech-X Corporation, http://www.txcorp.com/ products/OOPIC_Pro/) for the inter-electrode gap of $L=0.022 \mathrm{~m}$, tube radius $R=0.05 \mathrm{~m}$, rf voltage of $200 \mathrm{~V}$ and argon pressure of 0.1 Torr.

OOPIC Pro is a feature-rich 2D particle-in-cell (PIC) simulator. It is designed to model plasmas, beams of charged particles, externally generated electric and magnetic fields, and low-to-moderate density neutral gases, using a broad variety of boundary conditions. OOPIC Pro includes Monte Carlo collision (MCC) algorithms for modeling collisions of charged particles with a variety of neutral background gases, including such effects as ion-neutral charge exchange, elastic electron scattering, inelastic scattering due to electron impact excitation, and electron impact ionization. We chose electrostatic simulation field solver, the time step for the simulation was specified as $10^{-10} \mathrm{~s}$, the uniform gas temperature was set as $0.026 \mathrm{eV}$. Monte Carlo collisions were enabled, which means that collisions of particles with a background gas as well as the resulting ionization of the gas were taken into account. The initial uniform concentrations of the electrons and positive ions were $10^{15} \mathrm{~m}^{-3}$. Production of the secondary species modeled in OOPIC Pro employs a phenomenological model of the quantum processes that occur in the surface of materials. The ion induced

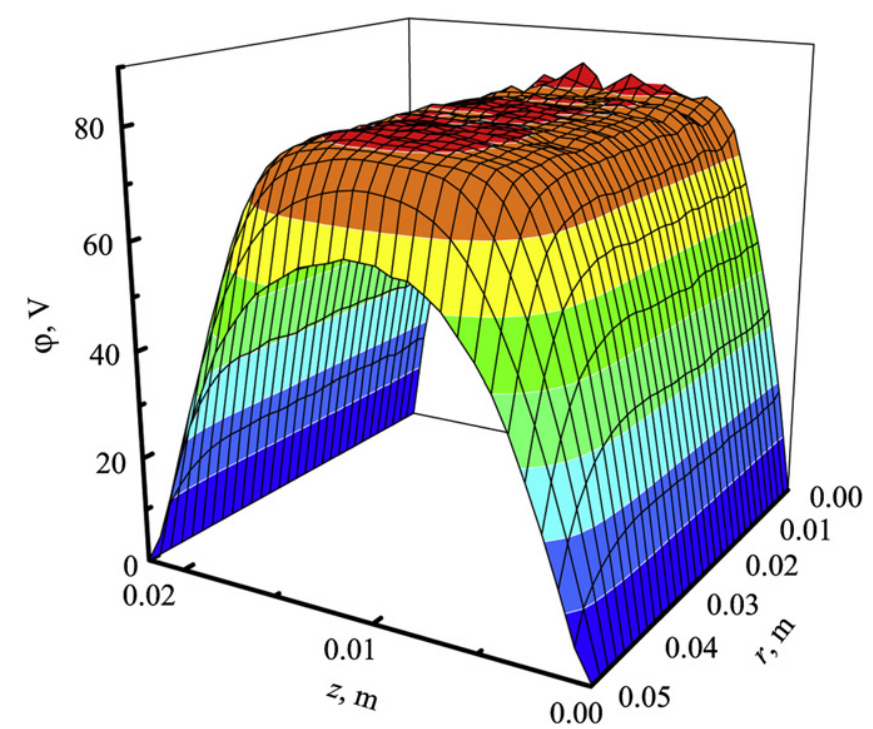

Fig. 12. Plasma potential distribution in rf discharge in argon at the pressure of 0.1 Torr and the rf voltage of $200 \mathrm{~V}$.

secondary-electron emission in our simulation was taken into account with a secondary emission coefficient of 0.1 .

The results of simulation are presented in Figs. 12-15 for the time moment when the instantaneous rf voltage at the driven electrode is close to zero. In Fig. 12 we depict the axial and radial distributions of plasma potential in the tube. It is clear from this figure that two near-electrode sheaths as well as a radial sheath near the tube wall are formed in the $\mathrm{rf}$ discharge. Rf voltage drop of about $20 \mathrm{~V}$ is available between the plasma and the tube wall (from $79 \mathrm{~V}$ in the plasma to $60 \mathrm{~V}$ near the tube wall). At the time moment when the rf electrode potential is maximum and all voltage drop is concentrated in one near-electrode sheath, in the region where this sheath is in contact with the tube wall the voltage drop across the radial sheath approaches $35 \mathrm{~V}$ whereas in the region of another sheath (being in the anode phase) the voltage drop across the radial sheath equals only about $5 \mathrm{~V}$. The axial rf electric field $E_{\mathrm{Z}}$ (Fig. 13) in the plasma volume does not exceed $10^{3} \mathrm{~V} / \mathrm{m}$, it approaches about $25 \mathrm{kV} / \mathrm{m}$ near the electrodes, but it is a little more than $11 \mathrm{kV} / \mathrm{m}$ near the tube wall. The distribution of the radial rf electric field

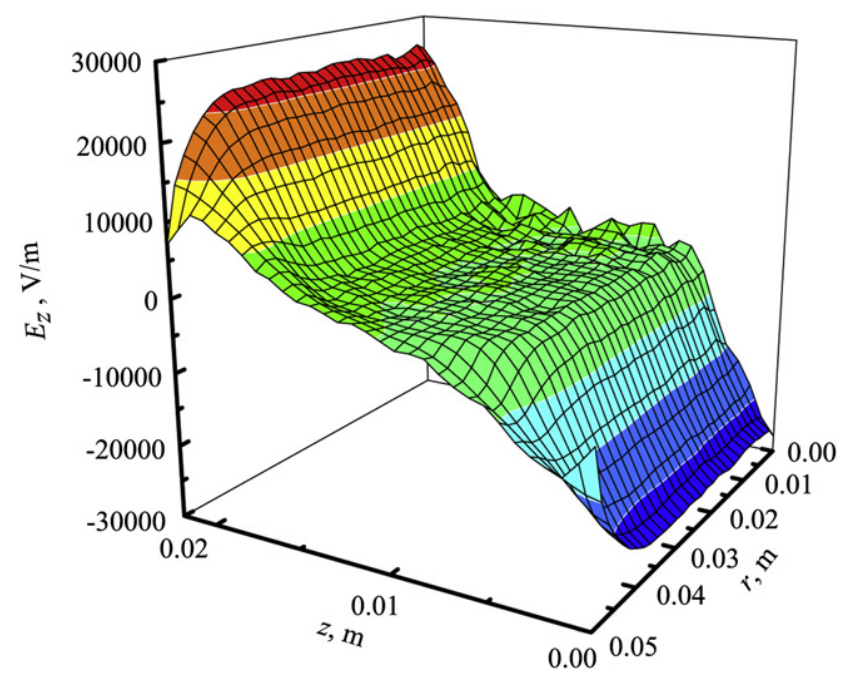

Fig. 13. Distribution of axial electric field in $\mathrm{rf}$ discharge in argon at the pressure of 0.1 Torr and the rf voltage of $200 \mathrm{~V}$. 


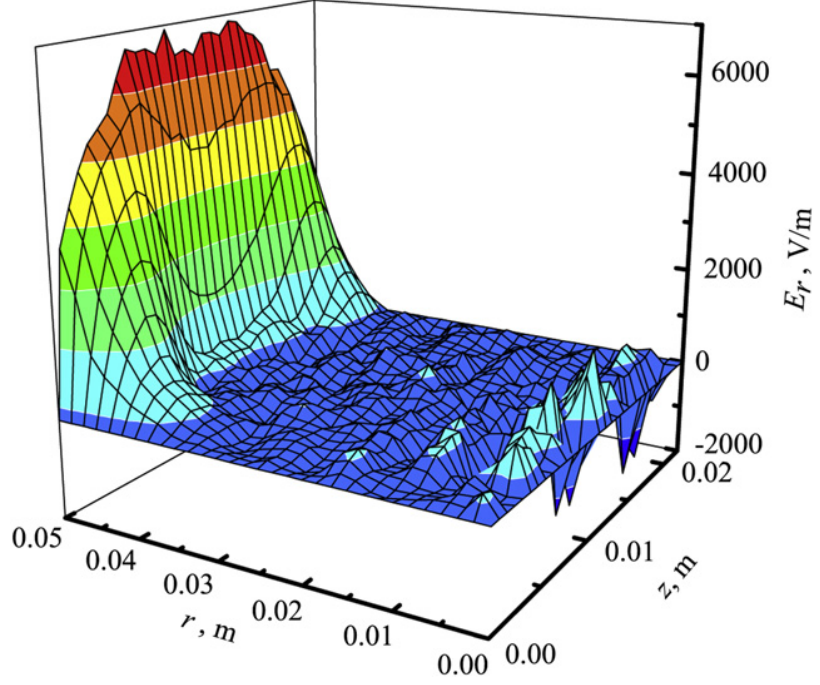

Fig. 14. Distribution of radial electric field in rf discharge in argon at the pressure of 0.1 Torr and the rf voltage of $200 \mathrm{~V}$.

(Fig. 14) deserves a special attention. In the central region of the discharge it comprises only several $\mathrm{V} / \mathrm{cm}$, but it grows almost to $7 \mathrm{kV} / \mathrm{m}$ near the tube wall. That is, the radial field in the central plane of the discharge may exceed the axial field considerably. The maximum of the radial rf field is observed just near the boundary of the near-electrode sheath which is in the cathode phase. Thus in this region an intense heating of those electrons occurs that are swept out of the near-electrode sheath by a moving boundary. Probably a portion of these electrons (the fastest ones) approaches the tube wall charging it negatively. The consideration of Fig. 14 (as well as of the radial field profiles for other instants of the rf field period) shows that the radial sheath thickness in the anode phase amounts only to $4-5 \times 10^{-3} \mathrm{~m}$ near the boundary of the nearelectrode sheath, whereas in the cathode phase it grows to 15$16 \times 10^{-3} \mathrm{~m}$ (the electric field strength in this discharge region grows to $8.5 \mathrm{kV} / \mathrm{m}$ ). Such a considerable expansion of the radial sheath sweeps the slow electrons out of the near-to-wall region into the plasma imparting a considerable energy to them. It explains why the blue glow in Fig. 7 for the nitrogen pressure of 0.1 Torr has the form of a broad ring, the brightest glow coming

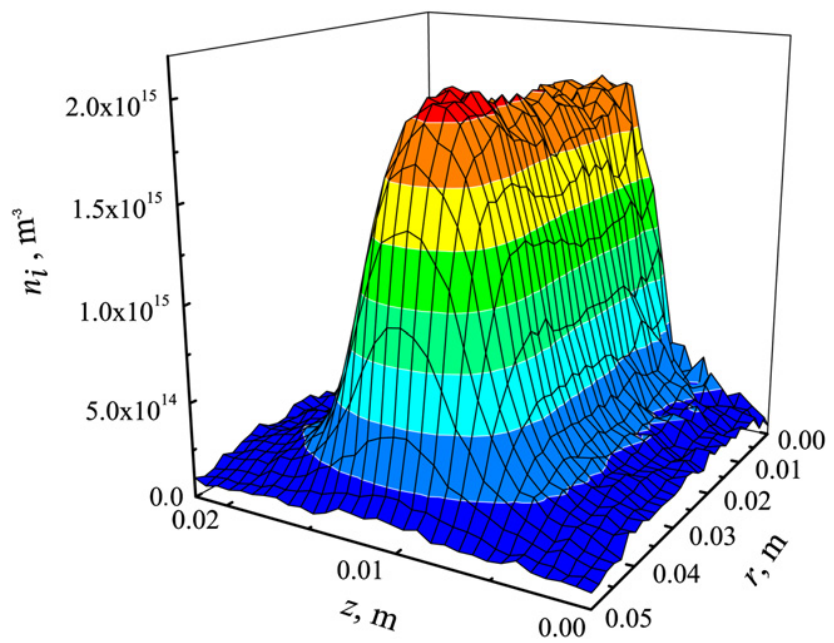

Fig. 15. Distribution of the ion concentration in the rf discharge in argon at the pressure of 0.1 Torr and the rf voltage of $200 \mathrm{~V}$.

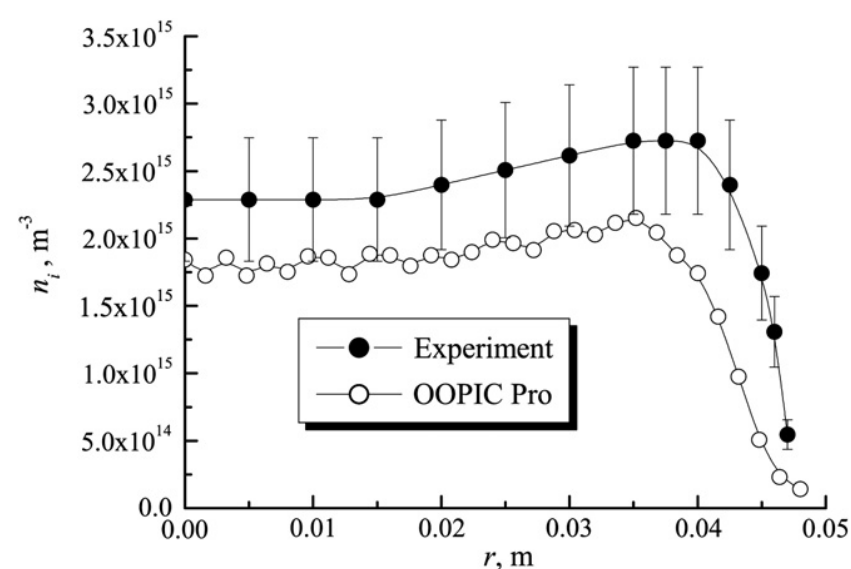

Fig. 16. Radial distribution of ion concentration in the rf discharge in argon at the pressure of 0.1 Torr and the rf voltage of $200 \mathrm{~V}$, inter-electrode gap $L=0.022 \mathrm{~m}$, tube radius $R=0.05 \mathrm{~m}$.

from the region near the boundaries of near-electrode sheaths whereas in the central plane of the discharge this glow is expressed weakly.

Fig. 15 shows the ion concentration profile in the rf discharge. It is clear from the figure that the ion concentration is small in nearelectrode sheaths as well as in the radial sheath. In the central plane the ion concentration grows counting from the discharge centre and it approaches its maximum near the boundary of the radial sheath. Note that this behaviour of the ion concentration agrees with our experimental data shown in Fig. $6 \mathrm{~b}$ for $\alpha$-mode of the rf discharge. The region of enhanced plasma concentration is, perhaps, created by electrons swept under expansion of the radial sheath and acquired the energy sufficient for ionizing gas molecules.

Fig. 16 demonstrates that the radial profile of ion concentration we registered in the central plane of the discharge with a Langmuir probe, as well as that calculated with OOPIC Pro code are in good agreement between themselves. The experimental data exceed those of modeling by $25 \%$ on the average. Both the registered and modeled profiles of ion concentration possess a maximum near the tube wall (35-40 $\times 10^{-3} \mathrm{~m}$ off the discharge axis).

The distribution of the radial electric field $E_{\mathrm{r}}$ presented in Fig. 14 corresponds to the time moment when the instantaneous if voltage at the potential electrode equals zero (see Fig. 12). During the rf field period $T$ this distribution changes considerably. Fig. 17 depicts the behaviour of the radial electric field near the tube wall $E_{\mathrm{rw}}$ (near the boundaries of near-electrode sheaths and in the discharge central plane) during a single period $T$. In the sheath cathode phase when the near-electrode sheath thickness achieves a maximum the radial electric field $E_{\mathrm{rw}}$ approaches $9 \mathrm{kV} / \mathrm{m}$. In the sheath anode phase when the electron cloud touches the electrode the $E_{\mathrm{rw}}$ value near the boundary of this sheath is minimum and it amounts to $6 \mathrm{kV} / \mathrm{m}$. In the discharge central plane the electric field $E_{\mathrm{rw}}$ oscillates within the range $7-8 \mathrm{kV} / \mathrm{m}$.

From formula (9) and radial profiles of $T_{\mathrm{e}}$ and $n_{\mathrm{i}}$ in Figs. 5, 6 and 16 it is easy to evaluate that the ambipolar field value does not exceed $1-2 \mathrm{kV} / \mathrm{m}$. It is much less than $E_{\mathrm{rw}}$ values in Fig. 17. Consequently the near-wall electric field $E_{\mathrm{rw}}$ is determined by the excess negative surface charge coming to the tube wall. Let us write the field $E_{\mathrm{rw}}$ in the form [38]

$E_{\mathrm{rw}}=\frac{e \cdot \Delta \sigma_{\mathrm{e}}}{\varepsilon_{0}}$,

where $\varepsilon_{0}$ is the permittivity of vacuum, $\Delta \sigma_{\mathrm{e}}=\sigma_{\mathrm{e}}-\sigma_{\mathrm{i}}$ is the negative charge concentration at the wall, $\sigma_{\mathrm{e}}$ and $\sigma_{\mathrm{i}}$ are the charge 


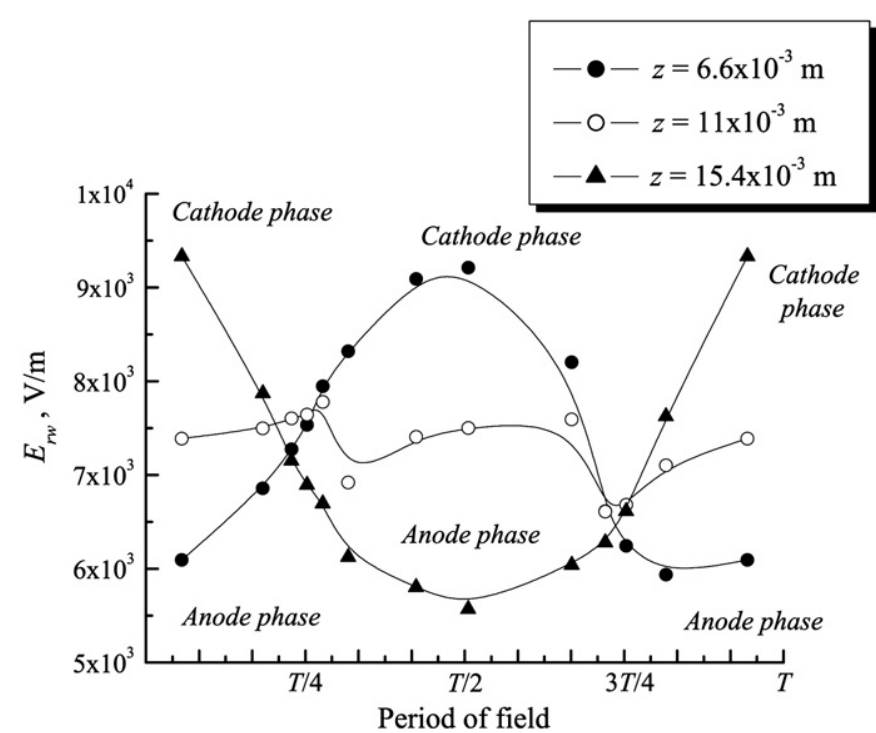

Fig. 17. Radial electric field $E_{\mathrm{rw}}$ variation near the tube wall for the near-electrode sheath boundaries $(z=6.6 \mathrm{~mm}$ and $z=15.4 \mathrm{~mm}$ ) and in a central plane during the $\mathrm{rf}$ field period. Argon, $p=0.1$ Torr, $U_{\mathrm{rf}}=200 \mathrm{~V}$, inter-electrode gap $L=0.022 \mathrm{~m}$, tube radius $R=0.05 \mathrm{~m}$.

concentrations at the wall. Fig. 17 and formula (6) permit to find that the negative charge $\Delta \sigma_{\mathrm{e}}$ concentration at the wall near the near-electrode sheath boundary in the cathode phase approaches $5 \times 10^{11} \mathrm{~m}^{-2}$, and in the anode phase it lowers to $\Delta \sigma_{\mathrm{e}} \approx 3.3 \times 10^{11} \mathrm{~m}^{-2}$. At the time moment, say, $\omega t=\pi$ in Fig. 17 one of the near-electrode sheaths (with the coordinate of its boundary equalling $z=6.6 \times 10^{-3} \mathrm{~m}$ ) is in the cathode phase, and another sheath (with the coordinate $z=15.4 \times 10^{-3} \mathrm{~m}$ ) is in the anode phase. Here we observe the strong decrease in the negative surface charge concentration $\Delta \sigma_{\mathrm{e}}$ at the wall with the coordinate $z$ varying. It indicates that just a near-electrode sheath being in the cathode phase at this moment of the rf period is a source of electron flow charging the tube wall.

\section{Conclusions}

Our paper is devoted to the investigation of the radial structure of the symmetric rf capacitive discharge. The uniformity of the plasma parameters radial distribution is of considerable interest in the processes of etching semiconductor materials and depositing films in technological chambers with a rf discharge. We registered the radial profiles of electron temperature and plasma concentration with a single Langmuir probe and studied the distribution of the glow intensity of the discharge plasma against the tube radius. An abrupt increase of electron temperature and glow intensity near the tube wall in the weak-current $\alpha$-mode of the rf discharge is observed. The radial distribution of the plasma concentration approaches a maximum near the radial sheath boundary. The rf discharge transition to the strong-current $\gamma$-mode leads to a more uniform distribution with a low electron temperature, to a weaker electric field and to the disappearance of the glow intensity peak near the tube wall. Besides, the 2D simulation of the rf discharge was made. The thickness of the radial sheath obtained with the 2D simulation experiences pulsations during the rf field period. The radial electric field leads probably to electron heating and plasma concentration increase near the boundary of the radial sheath in the $\alpha$-mode of the rf discharge. The results of the simulation we obtained are in a good agreement with our experimental data for the rf discharge in argon. The investigation made in this work permits us to determine the optimum conditions for uniform etching in the rf discharge.

\section{References}

[1] Booth JP. Plasma Sources Sci Technol 1999;8:249.

[2] Shul RJ, Pearton SJ. Handbook of advanced plasma processing techniques. Berlin: Springer; 2000.

[3] Sugawara M. Plasma etching: fundamentals and applications. Oxford: Oxford University Press; 1998.

[4] Lieberman MA, Lichtenberg AJ. Principles of plasma discharges and materials processing. Hoboken: Wiley; 2005.

[5] Perrin J. J Vac Sci Technol A 1998;16:278.

[6] Grill A. Cold plasma in materials technology: from fundamentals to applications. New York: IEEE Press; 1994.

[7] Korzec D, Rapp J, Theirich D, Engemann J. J Vac Sci Technol A 1994;12:369.

[8] Chen FF, Chang JP. Lecture notes on principles of plasma processing. Berlin: Plenum/Kluwer Publishers; 2002.

[9] Raizer YP, Shneider MN, Yatsenko NA. Radio-frequency capacitive discharges. Boca Raton, FL: CRC Press; 1995.

[10] Laroussi M. Plasma Processes Polym 2005;2:391.

[11] Reece Roth J. Industrial plasma engineering. In: Applications to nonthermal plasma processing, vol. 2. Bristol: IOP Publishing; 2001.

[12] Tsai JH, Wu C. Phys Rev A 1990;41:5626.

[13] Young FF, Wu Ch-H. Appl Phys Lett 1993;62:473.

[14] Young FF, Wu Ch-H. J Appl Phys 1993;74:839.

[15] Lieberman MA, Booth JP, Chabert P, Rax JM, Turner MM. Plasma Sources Sci Technol 2002;11:283.

[16] Overzet LJ, Hopkins MB. Appl Phys Lett 1993;63:2484.

[17] Lai WT, Hwang CJ, Wang AT, Yau JC, Liao JH, Chen LH, et al. In: Proceedings of the international symposium on dry process. Nagoya (Japan): Institute of Electrical Engineers; 2006. p. 109.

[18] Aoyagi K, Ishikawa I, Saito Y, Suganomata Sh. Jpn J Appl Phys 1996;35:6248.

[19] Takaki K, Koseki D, Fujiwara T. Appl Phys Lett 2002;80:1526.

[20] Volynets VN, Ushakov AG, Sung D, Tolmachev YN, Pashkovsky VG, Lee JB, et al. J Vac Sci Technol A 2008;26:406.

[21] Chen FF. Plasma Sources Sci Technol 2006;15:773.

[22] Jeon SJ, Eom GS, Kim JH, Choe W. Rev Sci Instrum 2002;73:277.

[23] Zakrzewski Z, Kopiczynski T. Plasma Phys 1974;16:1195.

[24] Tichy M, Sicha M, David P, David T. Contrib Plasma Phys 1994;34:59.

[25] Levitskii SM. Sov Phys Tech Phys 1957;2:887.

[26] Yatsenko NA. Sov Phys Tech Phys 1981;26:678.

[27] Belenguer Ph, Boeuf JP. Phys Rev A 1990;41:4447.

[28] Godyak VA, Khanneh AS. IEEE Trans Plasma Sci 1986;14:112.

[29] Godyak VA, Piejak RB, Alexandrovich BM. IEEE Trans Plasma Sci 1991;19:660.

[30] Vidaud P, Durrani SMA, Hall DK. J Phys D Appl Phys 1988;21:57.

[31] Lisovskiy VA. Tech Phys 1998;43:526.

[32] Lisovskiy VA, Yegorenkov VD. Vacuum 2004;74:19.

[33] Lisovskiy V, Booth J-P, Landry K, Douai D, Cassagne V, Yegorenkov V. Phys Plasmas 2006;13:103505.

[34] Lisovskiy V, Booth J-P, Landry K, Douai D, Cassagne V, Yegorenkov V. IEEE Trans Plasma Sci 2007;35:416.

[35] Raizer YP. Gas discharge physics. Berlin: Springer; 1991.

[36] Kimura T, Kano A, Ohe K. Phys Plasmas 1995;2:4659.

[37] Huang FY, Kushner MJ. J Appl Phys 1995;78:5909.

[38] Behnke JF, Bindemann T, Deutsch H, Becker K. Contrib Plasma Phys 1997;37:345. 